$06,11,19$

\title{
Влияние температуры обжига на размер зерен и электрокалорический эффект керамики титаната бария
}

\author{
(C) И.А. Старков ${ }^{1}$, А.С. Анохин ${ }^{1,2}$, И.Л. Мыльников ${ }^{1,2}$, М.А. Мишнев ${ }^{1}$, А.С. Старков ${ }^{1,2}$ \\ ${ }^{1}$ Санкт-Петербургский электротехнический университет „лЭТИ“, \\ Санкт-Петербург, Россия \\ ${ }^{2}$ Национальный исследовательский университет ИТМО, \\ Санкт-Петербург, Россия \\ E-mail: ferroelectrics@ya.ru
}

Поступила в Редакцию 21 сентября 2021 г.

В окончательной редакции 3 декабря 2021 г.

Принята к публикации 6 декабря 2021 г.

Проведено теоретическое исследование распределения поляризации и механических напряжений в сегнетоэлектрическом шаре, расположенном в неограниченном диэлектрическом пространстве. Шар покрыт диэлектрической и воздушной оболочкой. Внешнее электрическое поле вдали от шара считается однородным. Поляризация в шаре удовлетворяет нелинейному уравнению Ландау-Гинзбурга, в котором учитывается наличие электрострикции. Предполагается, что для шара малых размеров влияние локальных упругих напряжений на поляризацию можно заменить на их среднее значение по объему шара. При этом предположении получено распределение напряжений и электрического поля как в шаре, так и вне его. Получена зависимость температуры Кюри-Вейсса от радиуса шара. Найденное решение используется для моделирования свойств микрогранулированной керамики. Наряду с разработанной теоретической моделью была проведена серия экспериментов по измерению диэлектрической проницаемости и электрокалорического эффекта (ЭКЭ) для керамик $\mathrm{BaTiO}_{3}$, синтезированных при разных температурах. Изменение температуры при ЭКЭ проводилось прямыми методами. Наибольшее значение ЭКЭ достигнуто для керамики, синтезированной при $1350^{\circ} \mathrm{C}$. Величина изменения температуры при изменении электрического поля на $2 \mathrm{mV} / \mathrm{m}$ составила $\Delta T=0.42 \mathrm{~K}$. Полученные экспериментальные результаты показали возможность использования теоретической четырехфазной модели керамики для предсказания зависимости свойств керамики от температуры обжига.

Ключевые слова: сегнетоэлектрики, электрокалорический эффект, титанат бария $\left(\mathrm{BaTiO}_{3}\right)$, гранулированные среды, температура обжига, размер зерна.

DOI: $10.21883 /$ FTT.2022.04.52184.208

\section{1. Введение}

Керамические сегнетоэлектрические пленки и изготовленные на их основе многослойные структуры привлекают пристальное внимание благодаря возможностям их широкого применения в создании нового поколения устройств памяти, конденсаторов, пироэлектрических детекторов и т.д. (см., например, [1]). В последние годы область приложения подобных материалов расширилась за счет их использования в устройствах твердотельного охлаждения [2,3], основанных на электрокалорическом эффекте (ЭКЭ). Напомним, что под ЭКЭ понимается обратимое изменение тепловых свойств образца (температуры, энтропии, теплоемкости) при изменении электрического поля. Высокая диэлектрическая проницаемость сегнетоэлектриков, сильно зависящая от температуры, и наличие спонтанной поляризации приводит к существованию заметного ЭКЭ, достигающего максимальных значений вблизи температур фазовых переходов. Прогресс в создании твердотельного охладителя во многом сдерживается отсутствием легкодоступных материалов, обладающих большим ЭКЭ. Одним из возможных кандидатов в такие материалы являются керамические пленки на основе титаната бария $\mathrm{BaTiO}_{3}$, более дешевые и более простые в изготовлении, чем кристаллы.

Титанат бария является одним из наиболее распространенных сегнетоэлектриков с кристаллической структурой типа перовскита. Сегнетоэлектрические свойства $\mathrm{BaTiO}_{3}$ были обнаружены еще в 1944 г. в Физическом институте им. П.Н. Лебедева АН СССР Вулом и Гольдман [4]. При уменьшении температуры он испытывает 3 фазовых перехода: от параэлектрической кубической к сегнетоэлектрической тетрагональной, затем к орторомбической и, наконец, к ромбоэдрической фазе. Вблизи фазовых переходов кристаллический $\mathrm{BaTiO}_{3}$ обладает значительным ЭКЭ, достигающим $1.6 \mathrm{~K}$ при не очень больших напряжениях в $1 \mathrm{MV} / \mathrm{m}$ [5]. Для керамики ЭКЭ несколько меньше и составляет $1.3 \mathrm{~K}$ при напряжении $2 \mathrm{MV} / \mathrm{m}$ [6]. При комнатных температурах, где титанат бария испытывает фазовый переход от тетрагональной к орторомбической фазе, ЭКЭ весьма мал. Наибольшее изменение температуры при ЭКЭ в этом температурном диапазоне примерно в 4-5 раз меньше, чем в окрестности температуры Кюри-Вейсса. Так, при электрическом поле $2 \mathrm{MV} / \mathrm{m}$ величина $\Delta T$ не превышает $0.22 \mathrm{~K}$ по данным [7] и $0.1 \mathrm{~K}$ по данным [6,8,9]. 
Пленки, получаемые на сегодняшний день, как правило, являются поликристаллическими и представляют совокупность гранул размером от десятка нанометров до десятка микрометров. Согласно экспериментальным исследованиям, выполненным при помощи рентгеновского или нейтронного излучения $[10,11]$, каждая гранула окружена переходным слоем толщиной порядка $\sim 1 \mathrm{~nm}$ and $\varepsilon \sim 100$ (dead layer, passive layer). Наличие переходного слоя приводит к существенному изменению свойств сегнетоэлектрика. В частности, если размер гранулы меньше некоторого критического значения, оцениваемого в $10 \mathrm{~nm}$ для $\mathrm{BaTiO}_{3}$, то спонтанная поляризация в таких сегнетоэлектриках отсутствует $[10,12]$. Помимо диэлектрического слоя между гранулами имеются промежутки (поры), которые заполнены газообразной средой, содержащей смесь воздуха и пары пластификатора. Наличие пор приводит к уменьшению плотности керамики по сравнению с плотностью монокристаллического $\mathrm{BaTiO}_{3}$. Для сравнительно толстых пленок (толщиной более $1 \mu \mathrm{m}$ ) зависимость их свойств от толщины пропадает [12], и отличие пленок от керамики может быть описано при помощи граничных условий [13]. В дальнейшем ограничимся рассмотрением керамик как более простых в описании. Для описания пленок к выведенным ниже уравнениям следует просто добавить определенные граничные условия на поверхности пленки.

Исследование микро- и нанокристаллических керамик показывает сильную зависимость их физических свойств от условий синтеза и размера зерен $[6,8,9,12,14-16]$. Изменение температуры синтеза влияет не только на размеры зерен, но и на их форму, распределение, плотность керамики, пористость. Из имеющихся экспериментальных данных следует, что сегнетоэлектрическая керамика может рассматриваться как композит, образованный гранулами (зернами), покрытыми диэлектрической и воздушной оболочками, помещенными в матрицу (четырехфазная модель композита). В качестве формы включения может рассматриваться шар, сфероид или эллипсоид. Для сфероидальной формы гранулы решение электростатической задачи найдено в [17] при линейной зависимости поляризации от электрического поля. Для нелинейной модели Ландау-Гинзбурга (ЛГ) явные формулы можно получить только для электрического поля, параллельного одной из осей сфероида [18]. В теоретических разработках зависимость от упругих деформаций, как правило, отсутствует или предполагается известной [19]. Решение совместной задачи упругости и нелинейного уравнения ЛГ впервые получено в настоящей работе. Так как решение электростатической задачи достаточно подробно рассмотрено в [18], то основное внимание уделяется влиянию упругости на свойства керамики. Результаты теоретических исследований сравниваются с экспериментальными, полученными для керамики титаната бария.

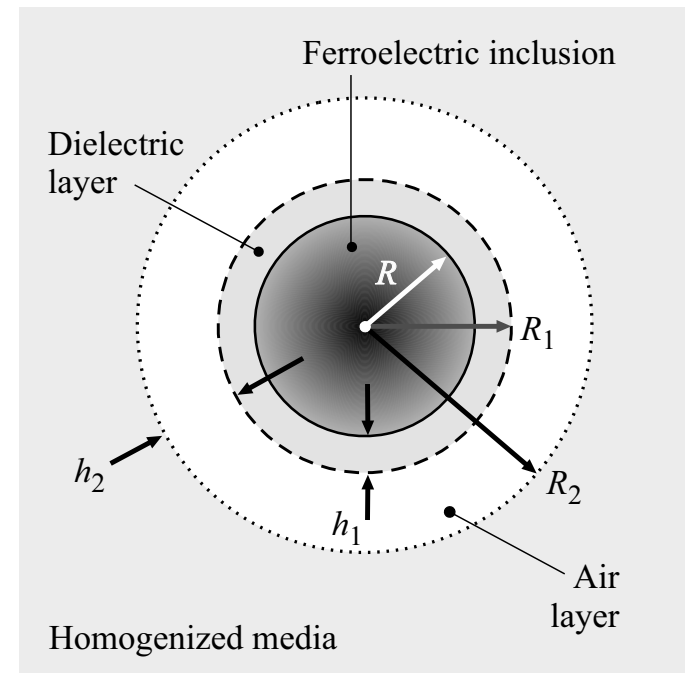

Рис. 1. Четырехфазная модель гранулы в сегнетоэлектрической керамике.

\section{2. Теоретическая модель}

\section{1. Функция распределения и физическая модель гранулы}

Будем моделировать керамику набором сегнетоэлектрических шаров. Радиус шара обозначим через $R$. Каждый шар покрыт двумя слоями радиусами $R_{1}$ и $R_{2}$ $\left(R<R_{1}<R_{2}\right)$ - диэлектрическим и воздушным, причем последний учитывает наличие пор между гранулами (см. рис. 1). Множество сегнетоэлектрических шаров помещены в диэлектрическую матрицу. Толщины слоев для рассматриваемого шара $h_{1}=R_{1}-R, h_{2}=R_{2}-R_{1}$ будем считать малыми по сравнению с радиусом $R$. Кроме того, толщину диэлектрического слоя $h_{1}$ будем считать не зависящей от $R$, что соответствует экспериментальным данным [10,11]. Зависимость толщины воздушного слоя от $R$ будем определять по относительной плотности $\rho$, где $\rho$ есть отношение плотности керамики $D$ к плотности кристаллического

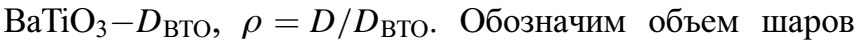
через $V_{\mathrm{g}}$, объем воздуха через $V_{\text {air }}$, а суммарный объ-

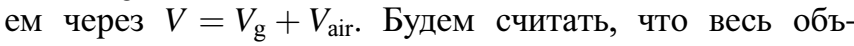
ем воздуха находится в поверхностных слоях. Масса керамики есть сумма масс шаров с диэлектрической оболочкой, плотность которых совпадает с плотностью чистого $\mathrm{BaTiO}_{3}$, и массы воздуха пор, которой пренебрегаем. Наличие воздушной массы приводит к уменьшению плотности керамики по сравнению с плотностью $\mathrm{BaTiO}_{3}$. Относительный суммарный объем шаров совпадает с относительной плотностью $\rho=V_{\mathrm{g}} / V$, а относительный суммарный объем воздушных слоев равен $V_{\text {air }} / V=1-\rho$. Предполагая, что весь воздух находится в воздушном слое, для его толщины, из элементарных геометрических соображений, получаем равенство $h_{2}(R)=\frac{1-\rho}{3 \rho} R$. Множитель $1 / 3$ получен при условии 
постоянства радиуса для всех гранул. Покажем, что этот множитель не зависит от функции распределения гранул по размерам $w(R)$. В предположении, что толщина слоя воздуха линейно зависит от $R, h_{2}=k R, k-$ некоторый коэффициент пропорциональности, средние объемы гранулы $\bar{V}_{\mathrm{g}}$ и воздуха $\bar{V}_{\text {air }}$ задаются интегралами

$$
\begin{gathered}
\bar{V}_{\mathrm{g}}=\frac{4 \pi}{3} \int_{0}^{\infty} w(R, L, \sigma) R^{5} \mathrm{~d} R, \\
\bar{V}_{\mathrm{air}}=4 \pi k \int_{0}^{\infty} w(R, L, \sigma) R^{5} \mathrm{~d} R .
\end{gathered}
$$

Постоянная $k$ определяется из отношения $\frac{V_{\text {air }}}{V_{\mathrm{g}}}=\frac{\bar{V}_{\text {air }}}{\bar{V}_{\mathrm{g}}}$ $=\frac{1-\rho}{\rho}$. Отсюда $k=(1-\rho) / 3 \rho$, и это значение не зависит от вида функции распределения.

Для нахождения электрического и упругого полей в керамике нужно знать их поведение в отдельно взятом шаре и функцию распределения $w(R)$ шаров по размерам. Функция $w(R)$ задается распределением гранул в момент начала обжига и временем обжига. Не вдаваясь в детали процесса обжига (этому процессу предполагается посвятить отдельную статью), будем предполагать, что $w(R)$ есть нормальное распределение. Точнее, $w(R)$ будем считать сферически симметричным трехмерным нормальным распределением, так как каждая гранула имеет три размера $\left(d_{1}, d_{2}, d_{3}\right)$. Трехмерное распределение получается из произведения трех нормальных одномерных распределений

$$
w_{0}\left(d_{i}, L_{i}\right)=\frac{1}{\sqrt{2 \pi} \sigma_{i}} \exp \left[-\frac{\left(d_{i}-L_{i}\right)^{2}}{2 \sigma_{i}^{2}}\right], \quad i=1,2,3
$$

где $L_{i}-$ наиболее вероятный размер гранулы по оси $x_{i}, \sigma_{i}-$ среднеквадратичное отклонение от $L_{i}$. Если предполагать равенство наиболее вероятных размеров $L_{1}=L_{2}=L_{3}=L / \sqrt{3}$ и среднеквадратичных отклонений $\sigma_{1}=\sigma_{2}=\sigma_{3}=\sigma$, то после интегрирования по углам в сферической системе координат, определяемой обычным образом по декартовой $\left(d_{1}, d_{2}, d_{3}\right),\left(R=\sqrt{d_{1}^{2}+d_{2}^{2}+d_{3}^{3}}\right)$, получаем явное выражение для $w(R)$

$$
\begin{aligned}
w(R) & \equiv w(R, L, \sigma) \\
& =\frac{2 R}{\sqrt{2 \pi} L \sigma R} \operatorname{sh}\left(\frac{R L}{\sigma^{2}}\right) \exp \left(-\frac{R^{2}+L^{2}}{2 \sigma^{2}}\right) .
\end{aligned}
$$

В дальнейшем под $w(R)$ будем понимать произвольный закон распределения, а под $w(R, L, \sigma)$ - трехмерное нормальное распределение. При отличающихся друг от друга значениях математических ожиданий $L_{1,2,3}$ формой гранулы является эллипсоид. Число гранул
$N\left(R_{\min }, R_{\max }\right)$, размер которых удовлетворяет неравенствам $R_{\min }<R<R_{\max }$, по определению функции распределения задается интегралом

$$
N\left(R_{\min }, R_{\max }\right)=\int_{R_{\min }}^{R_{\max }} w(R, L, \sigma) R^{2} \mathrm{~d} R
$$

Если среднеквадратичное отклонение достаточно мало $(3 \sigma<L$, правило $3 \sigma)$, то вклад гранул с радиусом $R<L-3 \sigma$ становится пренебрежимо малым. В этом случае с высокой степенью точности трехмерное распределение можно заменить на одномерное вида (2). Математическое ожидание $L$ и среднеквадратичное отклонение $\sigma$ определяются временем и температурой обжига $T_{\mathrm{g}}$. Параметры $\sigma, L$ можно определить по экспериментальным данным. При увеличении $T_{\mathrm{g}}$ происходит увеличение как $L$, так и $\sigma[6,8,9,12,14-16]$. Начальное распределение может быть нормальным, а может быть достаточно произвольным [20]. При увеличении времени обжига произвольное распределение, как правило, стремится к нормальному. Так для начального распределения, являющегося суммой двух нормальных с характерным размеров частиц 80 и $200 \mathrm{~nm}$, после $14 \mathrm{~h}$ обжига распределение близко к нормальному со средним размером $252 \mathrm{~nm}$ [21]. Сравнение с экспериментальными данными показывает, что в некоторых случаях необходимо использовать трехмерное распределение (3) [15], а в некоторых можно ограничиться его одномерным приближением вида (2). В дальнейшем будем использовать распределение (3) как более общее.

\section{2. Постановка задачи и основные уравнения}

Перейдем теперь к описанию отдельного шара радиуса $R$. Будем исследовать распределение полей в одиночном шаре, который находится во внешнем электрическом поле $\mathbf{E}_{\text {ext. }}$ На больших расстояниях от шара это поле считается однородным и направленным параллельно оси аппликат. Предполагается, что помимо электрического поля со стороны соседних шаров действует гидростатическое давление $p$. Это давление может происходить из-за рассогласования параметров кристаллических решеток, наличия спонтанной деформации, электрострикционных эффектов в матрице, различия коэффициентов теплового расширения для фаз.

Для описания электрического и упругого полей будем использовать электрический потенциал $\varphi$, поляризацию $P$ и упругие смещения $u_{i}, i=1,2,3$. По ним определяются напряженность электрического поля $\mathbf{E}=-\nabla \varphi$ и упругие напряжения $u_{i j}=\left(u_{i, j}+u_{j, i}\right) / 2$. Здесь и далее индекс после запятой означает дифференцирование по соответствующей переменной. В дальнейшем будем предполагать, что поляризация и электрическое поле в шаре направлены параллельно внешнему электрическому полю, и их можно считать скалярными величинами. 
При этом предположении связь поляризации и напряженности в шаре задается обобшенным уравнением Ландау-Гинзбурга [22]:

$$
E=a P+b P^{3}+c P^{5}-2 P\left[q_{12}\left(u_{11}+u_{22}\right)+q_{11} u_{33}\right] .
$$

Здесь $a, b, c$ есть коэффициенты ЛГ, $q_{i j}$ - электрострикционные коэффициенты. Причем от температуры $T$ зависит только коэффициент $a=a_{0}\left(T-T_{\mathrm{CW}}\right)$, где $a_{0}=1 / \varepsilon_{0} C, T_{\mathrm{CW}}$ - температура Кюри-Вейсса, $C$ постоянная Кюри и $\varepsilon_{0}$ есть электрическая постоянная. Уравнение (5) справедливо, если поляризация и электрическое поле имеют одинаковое направление. Для его исследования необходимо найти значения тензора деформации и напряженность электрического поля по заданному значению $E_{\text {ext }}$.

Вначале выпишем связь тензора напряжений $\sigma_{i j} \mathrm{c}$ тензором деформаций (закон Гука) при наличии электрострикции [22]:

$$
\begin{gathered}
\sigma_{11}=c_{11} u_{11}+c_{12} u_{22}+c_{12} u_{33}-q_{12} P^{2}, \\
\sigma_{22}=c_{12} u_{11}+c_{11} u_{22}+c_{12} u_{33}-q_{12} P^{2}, \\
\sigma_{33}=c_{12} u_{11}+c_{12} u_{22}+c_{11} u_{33}-q_{11} P^{2}, \\
\sigma_{23}=2 c_{44} u_{23}, \quad \sigma_{13}=2 c_{44} u_{13}, \quad \sigma_{12}=2 c_{44} u_{12},
\end{gathered}
$$

где $c_{11}, c_{12}, c_{44}$ есть упругие постоянные в матричных обозначениях (Фойгта). В дальнейшем ограничимся случаем изотропной среды, когда есть только 2 независимые упругие постоянные. Отметим, что для уточнения модели в правую часть соотношения (6) для $\sigma_{i i}$ можно добавить слагаемые вида $\beta\left(T-T_{0}\right)$, определяющие термоупругие напряжения. Здесь $T_{0}-$ температура, при которой термоупругие деформации отсутствуют, а коэффициент $\beta$ связан с коэффициентом теплового расширения $\alpha$ соотношением $\beta=-\left(c_{11}+2 c_{12}\right) \alpha$. Помимо термоупругих слагаемых можно учитывать и деформации, вызванные рассогласованием кристаллических решеток. Важным моментом является то, что термоупругие слагаемые внутри шара есть постоянные величины. Для шара ограничимся только электрострикционными слагаемыми, а для матрицы объединим вышеперечисленные факторы в гидростатическое давление $p$.

Введенные величины должны удовлетворять стандартным уравнениям электростатики и упругости [23]:

$$
\operatorname{div}\left(\varepsilon_{0} E+P\right)=0, \quad \sigma_{i j, j}=0 .
$$

Отсюда сразу следует, что напряженность электрического поля $E_{3}$ и поляризация $P_{3}$ в шаре не только направлены по одной оси, но и постоянны. Это позволяет рассматривать задачи нахождения поляризации и деформаций независимо друг от друга.

\section{3. Приближенное решение}

Из предположения об однородности поляризации в уравнениях (6) вытекает однородность деформации. Общая однородная деформация может быть представлена в виде суммы однородного всестороннего (гидростатического) растяжения (или сжатия) и однородного сдвига [24]. Наличие сдвига приводит к появлению дополнительных компонент в электрическом поле и поляризации, т.е. к нарушению предположения об однородности поляризации. Деформация сдвига пропорциональна $R^{-2}$ и, кроме того, ее среднее значение по шару есть 0 [24]. Ввиду этого для микрогранул $(R>200 \mathrm{~nm})$ этой частью деформации можно пренебречь и рассматривать только всестороннее сжатие. Для этого перейдем в сферическую систему координат $(r, \vartheta, \varphi)$ с центром в начале шара. В силу сферической симметрии как нагрузки, так и исследуемой системы, единственной отличной от нуля компонентой вектора смещения будет радиальная $u_{r}$.Ненулевые компоненты тензора деформации при этом имеют вид $u_{r r}=u_{r, r}, u_{\vartheta \vartheta}=u_{\varphi \varphi}=u_{r} / r$ [24]. На границах разделов сред (при $r=R, R_{1}, R_{2}$ ) должны быть непрерывны смещение $u_{r}$ и $\sigma_{r r}$. Общее решение уравнений упругости в случае гидростатических сил имеет вид [24]:

$$
u_{r}=B_{1} r+B_{2} \frac{1}{r^{2}}
$$

где $B_{1,2}$ - произвольные постоянные. Так как у нас имеется 4 среды, то имеется 8 постоянных, из которых 2 находятся сразу в силу требования ограниченности смещения в центре шара и на бесконечности. В результате имеем 6 неизвестных, для которых можно выписать систему линейных уравнений, вытекающую из граничных условий. К сожалению, анализ полученного решения затруднен в силу громоздкости полученных выражений, поэтому воспользуемся малостью толщин слоев и упростим граничные условия. Для этого применим матричный метод [25], который позволяет исключить из рассмотрения тонкие слои и заменить их наличие на приближенные граничные условия. Составим столбец $X$ из величин, непрерывных на границах различных сред, $X=\left(u_{r}, \sigma_{r r}\right)^{\operatorname{tr}}$. Символ $t r$ означает транспонирование. Соотношения (6) (при $P=0$ ) и уравнения (7) в сферической системе координат переписываются в виде [24]:

$$
\sigma_{r r}=c_{11} u_{r r}+2 c_{12} \frac{u_{r}}{r}
$$

$$
\begin{gathered}
\sigma_{\vartheta \vartheta}=\sigma_{\varphi \varphi}=c_{12} u_{r r}+\left(c_{11}+c_{12}\right) \frac{u_{r}}{r}, \\
\frac{\mathrm{d} \sigma_{r r}}{\mathrm{~d} r}+\frac{1}{r}\left(2 \sigma_{r r}-\sigma_{\vartheta \vartheta}-\sigma_{\varphi \varphi}\right)=0 .
\end{gathered}
$$


Из уравнений (9) находим выражение для производной от $X$ по $r$

$$
\begin{gathered}
\frac{\mathrm{d} X}{\mathrm{~d} r}=M X, \\
M(r) \equiv\left(\begin{array}{cc}
-\frac{2 c_{12}}{r c_{11}} & \frac{1}{c_{11}} \\
\frac{2}{r^{2}}\left(c_{11}+c_{12}-\frac{2 c_{12}^{2}}{c_{11}}\right) & -\frac{2}{r}\left(1-\frac{c_{12}}{c_{11}}\right)
\end{array}\right) .
\end{gathered}
$$

Так как функция $X(r)$ является непрерывной и дифференцируемой, то ее значения на границах тонких слоев связаны приближенным равенством

$$
X\left(R_{2}\right)=\left(I+M_{1}(R) h_{1}+M_{2}(R) h_{2}+O\left(h_{1}^{2}+h_{2}^{2}\right)\right) X(R) .
$$

Здесь $I-$ единичная матрица размером $2 \times 2$, а матрица $M_{1}(r)$ имеет вид (10), в котором значения упругих постоянных взято для первого слоя, а в матрицу $M_{2}(r)$ входят только упругие коэффициенты для второго слоя. Изменением координаты $r$ в слоях пренебрегаем. Более точные формулы, содержащие $\int M_{1,2}(r) \mathrm{d} r$, приведены в [18]. Равенство (11) позволяет исключить оба слоя из рассмотрения и заменить их влияние на приближенные граничные условия, тем более точные, чем меньше толщина слоев. Для выписывания упрощенных граничных условий необходимо учесть наличие слагаемых, приводящих к появлению деформации. В шаре это среднее значение электрострикционных слагаемых $-\bar{q} P^{2}$, где $\bar{q}=\left(q_{11}+2 q_{12}\right) / 3$ есть среднее значение электрострикционных коэффициентов. В матрице деформация возникает из-за присутствия давления $p$. Добавляя в равенство (11) указанные величины приходим к граничному условию

$$
\begin{gathered}
X\left(R_{2}\right)=\left(I+M_{1}(R) h_{1}+M_{2}(R) h_{2}\right) X(R)+F, \\
F=\left(\begin{array}{c}
0 \\
\bar{q} P^{2}+p
\end{array}\right) .
\end{gathered}
$$

Теперь в соответствии с общим решением (8) отыскиваем смещение в шаре в виде $u_{r}=A^{i} r$, а в матрице - в виде $u_{r}=A^{m} R^{3} / r^{2}$, где $A^{i}$ и $A^{m}$ - искомые постоянные, имеющие размерность длины. Верхний индекс указывает на принадлежность величины включению $(i)$ или матрице $(m)$. При подстановке указанных выражений в граничные условия (12) и вычислении $\sigma_{r r}$ получаем систему для определения постоянных $A^{i, m}$

$$
\begin{array}{r}
A^{m}\left(\begin{array}{c}
R \\
-3 c_{11}^{m}+2 c_{12}^{m}
\end{array}\right)=A^{i}\left(I+M_{1}(R) h_{1}\right. \\
\left.+M_{2}(R) h_{2}\right)\left(\begin{array}{c}
R \\
c_{11}^{i}+2 c_{12}^{i}
\end{array}\right)+F .
\end{array}
$$

Несмотря на возможность получения точного решения системы (13) выпишем приближенное решение, полученное из точного разложением в ряд по малому параметру $\left(h_{1}+h_{2}\right) / R$

$$
\begin{gathered}
u_{i}=A^{i} r, \quad u_{r r}=u_{\vartheta \vartheta}=u_{\varphi \varphi}=A^{i}, \\
A^{i}=\left(A_{0}+A_{1} \frac{h_{1}}{R}+A_{2} \frac{h_{2}}{R}\right)\left(p+\bar{q} P^{2}\right) .
\end{gathered}
$$

Постоянные $A_{j}, j=0,1,2$ определяются только упругими свойствами фаз (коэффициентами матриц $M_{1}(R)$ и $\left.M_{2}(R)\right)$ и не зависят от радиуса $R$, например,

$$
A_{0}=-\frac{1}{c_{11}^{i}+2 c_{12}^{i}+2\left(c_{11}^{m}-c_{12}^{m}\right)} .
$$

Формулы для постоянных $A_{1,2}$ не выписываем в силу их громоздкости. Отметим, что в $A_{1}$ входят только упругие постоянные включения, матрицы и диэлектрического слоя. Отсюда следует, что коэффициент $A_{1}$ слабо зависит от условий получения керамики. Наоборот, коэффициент $A_{2}$ определяется свойствами воздушного слоя, которые сильно зависят от условий спекания. Полученные значения для смещения позволяют найти компоненты тензора деформации и получить следующее уравнение для поляризации в шаре

$$
E=\hat{a} P+\hat{b} P^{3}+c P^{5},
$$

где модифицированные коэффициенты ЛГ $\hat{a}, \hat{b}$ имеют вид

$$
\begin{aligned}
& \hat{a}=a-2 p\left(2 q_{12}+q_{11}\right)\left(A_{0}+A_{1} \frac{h_{1}}{R}+A_{2} k\right), \\
& \hat{b}=b-2 \bar{q}\left(2 q_{12}+q_{11}\right)\left(A_{0}+\frac{A_{1}}{R}+A_{2} k\right) .
\end{aligned}
$$

Величина $k=h_{2} / R=(1-\rho) / 3 \rho$ определяется относительной плотностью керамики $\rho$. При решении электростатической задачи получаем $E=\varkappa E_{\text {ext }}$, где коэффициент $\varkappa$ определяется диэлектрическими постоянными слоев и матрицы [18].

Из формулы (17) следует, что изменение коэффициентов ЛГ $\hat{a}-a$ и $\hat{b}-b$ при изменении радиуса $R$ пропорционально друг другу. Отметим также, что исходное уравнение для сегнетоэлектрического материала шара в уравнении состояния содержало только один зависящий от температуры коэффициент - $а$. Для керамики зависящим от $T$ следует считать два коэффициента в (16).

Из уравнения (16) вытекает следующая зависимость температуры фазового перехода $T_{\mathrm{PT}}$ от размера зерен

$$
T_{\mathrm{PT}}=T_{\mathrm{CW}}-2 \varepsilon_{0} C p\left(2 q_{12}+q_{11}\right)\left(A_{0}+A_{1} \frac{h_{1}}{R}+A_{2} k\right) .
$$

При малых (менее $200 \mathrm{~nm}$ ) размерах гранул в зависимости (18) главную роль играет слагаемое, пропорциональное $R^{-1}$. В результате получаем обратную пропорциональность $T_{\mathrm{PT}}$ размеру зерен [12]. Причем коэффициент пропорциональности слабо зависит от 
условий синтеза керамики. В [26] при исследовании керамики $0.9 \mathrm{~Pb}\left(\mathrm{Mg}_{1 / 3} \mathrm{Nb}_{2 / 3}\right)_{3}-0.1 \mathrm{PbTi}_{3}$ было найдено, что $T_{\mathrm{PT}}$ имеет максимум при определенном размере зерен. Для керамического титаната бария наличие максимума $T_{\mathrm{PT}}$ было обнаружено в [27]. Существование максимума температуры фазового перехода может быть объяснено в рамках предложенной модели. Экстремальность $T_{\mathrm{PT}}$ связана с существованием корней уравнения

$$
A_{1} \frac{h_{1}}{R^{2}}=A_{2} \frac{\mathrm{d} k}{\mathrm{~d} R}, \quad A_{1} \frac{h_{1}}{R^{2}}=-\frac{A_{2}}{3 \rho^{2}} \frac{\mathrm{d} \rho}{\mathrm{d} R},
$$

т. е. с поведением плотности керамики. Для существования корней уравнения (19) необходимо убывание плотности с ростом среднего размера гранул, т.е. с ростом температуры обжига $T_{\mathrm{g}}$. Такая ситуация возможна при достаточно больших температурах обжига, превышающих $1300^{\circ} \mathrm{C}$. Например, в [6] плотность керамики титаната бария имеет неявно выраженный максимум при $T_{\mathrm{g}}=1350^{\circ} \mathrm{C}$. Значит, при $T_{\mathrm{g}}>1350^{\circ} \mathrm{C}$ возможно появление гранул с максимальной температурой фазового перехода.

Полученные формулы позволяют рассчитывать основные характеристики керамики: поляризацию, диэлектрическую проницаемость, теплоемкость, пироэлектрический и электрокалорический коэффициенты, ЭКЭ. Например, среднее значение поляризации определяется по формуле

$$
P=\int w(R, L, \sigma) P(R) \mathrm{d} R .
$$

Здесь $P(R)$ - средняя поляризация в шаре $B_{2}$, в который входят оба слоя. Для локального пироэлектрического коэффициента $\partial P / \partial T$ в сегнетоэлектрике из (16) получаем следующую формулу

$$
\frac{\partial P}{\partial T}=\frac{\hat{a}_{T} P+\hat{b}_{T} P^{3}}{\hat{a}+3 \hat{b} P^{2}+5 c P^{4}},
$$

где

$$
\hat{a}_{T}=\frac{\mathrm{d} \hat{a}}{\mathrm{~d} T}, \quad \hat{b}_{T}=\frac{\mathrm{d} \hat{b}}{\mathrm{~d} T} .
$$

есть производные от модифицированных коэффициентов ЛГ по температуре. ЭКЭ для одиночного шара находится по известному пироэлектрическому коэффициенту при интегрировании по $B_{2}$ формулы для локального ЭКЭ [3]

$$
\mathrm{d} T=-\frac{T}{C V_{2}}\left(\int_{B_{2}} \frac{\partial P}{\partial T} \mathrm{~d} V\right) \mathrm{d} E .
$$

Здесь $C$ - теплоемкость системы (шара $B_{2}$ ), которая определяется как сумма теплоемкостей шара и окружающих его слоев.

Уравнение (23) записано в дифференциальной форме. В интегральной форме это уравнение принимает вид

$$
\Delta_{R} T=-\int_{E_{1}}^{E_{2}} \frac{T}{C V_{2}}\left(\int_{B_{2}} \Pi \mathrm{d} V\right) \mathrm{d} E .
$$

Здесь $\Delta_{R} T-$ изменение температуры в отдельной грануле радиуса $R$, вызванное изменением электрического поля от значения $E_{1}$ до значения $E_{2}$. Изменение температуры всего образца $\Delta T$ для произвольного закона распределения гранул по размерам $w(R)$, записывается в виде

$$
\Delta T=-\int_{R} \int_{E_{1}}^{E_{2}} \frac{T}{C V_{2}}\left(\int_{B_{2}} \Pi \mathrm{d} V\right) w(R) \mathrm{d} E \mathrm{~d} R .
$$

Отметим, что по сравнению с известными формулами для ЭКЭ [3], в уравнения (23), (24) вошло дополнительное интегрирование по объему гранулы, а в основной формуле ЭКЭ (25) имеется еще и интегрирование по размеру гранул $R$. Формула (25) является универсальной и не зависит от вида закона распределения $w(R)$ и пироэлектрического коэффициента П.

Таким образом, для нахождения изменения температуры керамики при изменении электрического поля необходимо знать функцию распределения гранул по размерам $w(R)$, пироэлектрический коэффициент П и теплоемкость гранулы $C$. Теплоемкость гранулы легко определяется по известным теплоемкостям титаната бария/воздуха и значению относительной плотности $\rho$, которое находится экспериментально. В качестве функции распределения можно выбрать нормальное распределение $w(R, L, \sigma)(3)$, в котором средний размер гранул $L$ и среднеквадратичное отклонение $\sigma$ нетрудно определить по экспериментальным данным. Основную сложность представляет нахождение пирокоэффициента П, для определения которого и используется разработанная выше четырехфазная модель. Величина П определяется упругими и электрическими свойствами сегнетоэлектрического ядра (шара), диэлектрического и воздушного слоев и их геометрическими параметрами. Для титаната бария и воздуха эти свойства известны [22]. Толщина воздушного слоя задается относительной плотностью $\rho$. Диэлектрический слой исследован намного хуже. Главной проблемой является нахождение температурной зависимости его диэлектрической проницаемости, так как малая толщина слоя не позволяет отделить его свойства от свойств ядра. Ввиду этого в данной работе используется предположение об отсутствии зависимости свойств диэлектрического слоя от температуры, которое может приводить к заметной разнице между теоретическими и экспериментально полученными зависимостями.

Единственной характеристикой, которая не может быть получена из уравнения состояния (16), является проводимость, поведение которой принято описывать в рамках теории Хейванга [28]. Проводимость достаточно сильно зависит от свойств переходных слоев между гранулами, поэтому для объяснения ее свойств следует уточнить модель диэлектрического и воздушного слоев, включив в нее распределение зарядов. 


\section{3. Экспериментальные измерения}

Для исследования зависимости свойств сегнетоэлектрика от температуры обжига была проведена серия экспериментов с керамикой титаната бария. Образцы изготавливались методом высокотемпературного спекания. Синтез производился в 2 стадии (two-step sintering): исходно измельчались и смешивались оксид титана и барий углекислый, далее производился длительный обжиг при температуре, достаточной для активного протекания реакции между твердыми фазами компонентов $\left(1200^{\circ} \mathrm{C}\right.$ в течение двух часов), затем полученные порошки повторно измельчались и смешивались. Полученные шихты перемешивались с органическим пластификатором (лак на основе акриловой смолы), помещались в прессформу и спрессовывались при давлении $100 \mathrm{MPa}$, внутренний диаметр пресс-форм составлял $12 \mathrm{~mm}$. Затем сырые заготовки синтезировались при высокой температуре, а полученные керамические образцы утонялись до толщины, равной $500 \mu \mathrm{m}$. На полированную поверхность изготовленных образцов наносились электроды, которые изготавливались методом вжигания проводящей серебряно-палладиевой пасты. На всех стадиях синтеза скорость изменения температуры в печи не превышала $0.07 \mathrm{~K} / \mathrm{s}$. При обжиге сырых заготовок программа нагрева предусматривала снижение скорости нагрева до $0.03 \mathrm{~K} / \mathrm{s}$ при температурах $500-700^{\circ} \mathrm{C}$ для минимизации снижения плотности керамики вследствие интенсивного испарения пластификатора. Время окончательного обжига варьировалось в пределах от 1 до $3 \mathrm{~h}$, а температура $T_{\mathrm{g}}$ менялась от $1200^{\circ} \mathrm{C}$ до $1400^{\circ} \mathrm{C}$.

Фазовый состав изготовленных образцов был исследован методом рентгено-структурного анализа. На рис. 2 приведены сравнительные рентгено-дифракционные спектры образцов титаната бария, спеченных при различной температуре. Полученные спектры соответствуют спектру титаната бария в тетрагональной фазе. В работе [29] различие в электрофизических свойствах титаната бария, спеченного при разных температурах, объяснялось именно различием в фазовом составе. Проведенные рентгенографические исследования не выявили различия в фазовом составе образцов, синтезированных при температурах начиная с $1325^{\circ} \mathrm{C}$, следовательно, куда более вероятным представляется, что различие в электрофизических свойствах связано с микроструктурой образцов, в частности, с размером зерна.

Изображения скола образцов, полученные методом растровой электронной микроскопии (scanning electron microscopy), представлены на рис. 3. Средние размеры гранул изменялись при этом от 2 до $33 \mu \mathrm{m}$. Плотность керамических образцов $D$ определялась методом гидростатического взвешивания с помощью лабораторных весов PX-224 OHAUS Pioneer. Определение кажущейся плотности проводилось способом гидростатического взвешивания свежерасколотых кусков образцов керамических материалов, предварительно насыщенных жидкостью. Для насыщения способом кипячения образцы

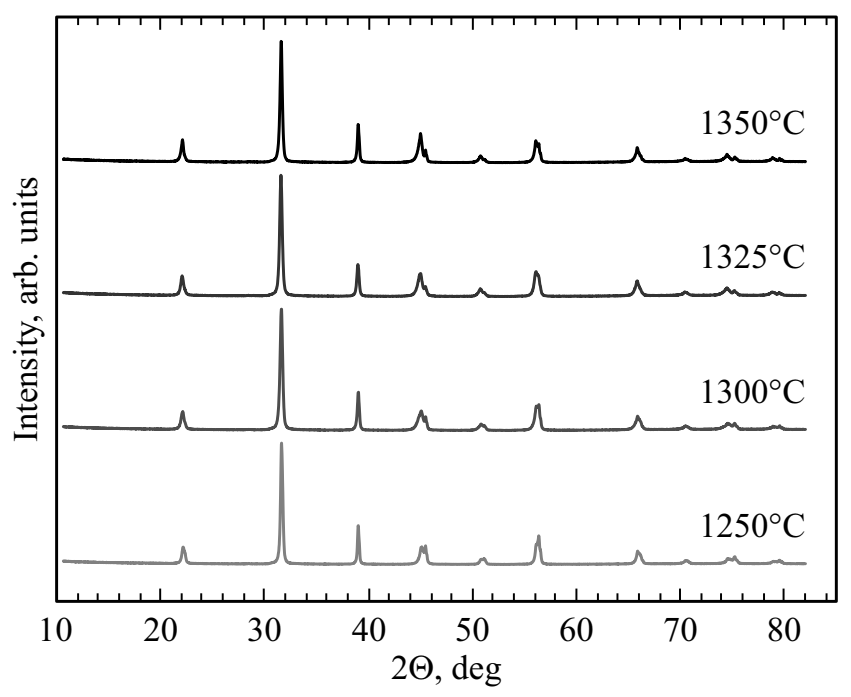

Рис. 2. Рентгено-дифракционные спектры образцов титаната бария при комнатной температуре.

были высушены в сушильном шкафу при температуpe $115 \pm 5^{\circ} \mathrm{C}$ до достижения постоянной массы. Гидростатическое взвешивание образцов после кипячения производилось в дистиллированной воде. Полученные экспериментальные зависимости $L$ и результаты измерения плотности керамики при различных температурах обжига синтеза изображены на рис. 4.

Изготовленные образцы были подвергнуты исследованию электрофизических и теплофизических свойств. Измерение температурных зависимостей диэлектрической проницаемости и тангенса угла диэлектрических потерь $\varepsilon(E, T), \tan \delta(E, T)$ производилось с помощью цифрового измерителя иммитанса Agilent E4980A. Режим изменения температуры при этом задавался термостатом Julabo $32 \mathrm{ME}$. Непосредственно измеряемой величиной при этом являлась электрическая емкость. В используемых пределах измерений погрешность ее измерения составляла менее $0.1 \mathrm{pF}$, относительная погрешность измерений составляла $0.05 \%$. В качестве измерительного сигнала использовался сигнал амплитудой равной $1 \mathrm{~V}$. В связи с достаточно заметной дисперсией диэлектрической проницаемости, характерной для сегнетоэлектрических материалов, измерения производились при различных частотах синусоидального сигнала в диапазоне от $100 \mathrm{~Hz}$ до $1 \mathrm{MHz}$. Результаты измерения диэлектрической проницаемости представлены на рис. $5, a$. На рис. 5, $b$ приведена зависимость диэлектрических потерь от температуры.

Измерение температурных, полевых и частотных зависимостей электрокалорического эффекта осуществлялось методом прямых измерений адиабатического изменения температуры с помощью прецизионных платиновых терморезистивных датчиков M213 Pt-100. Точность температурных измерений составляла $\pm 2 \mathrm{mK}$. Измерения проводились вблизи фазового перехода между тетрагональной и ромбоэдрической фазами, т.е. при 

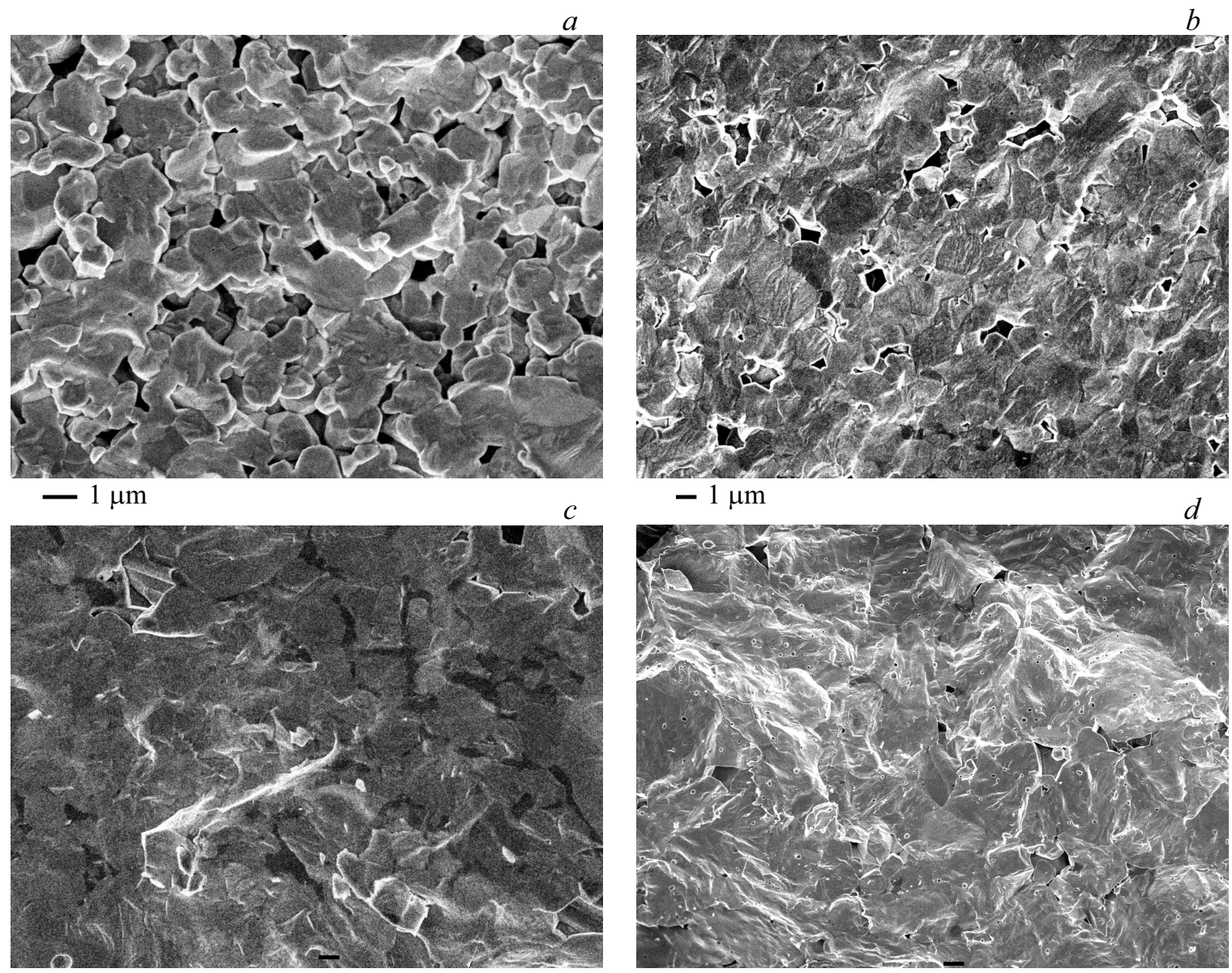

$-1 \mu \mathrm{m}$

$-1 \mu \mathrm{m}$

Рис. 3. Микроструктурный анализ образцов керамики титаната бария, отожженных при $T_{\mathrm{g}}=(a) 1250^{\circ} \mathrm{C},(b) 1300^{\circ} \mathrm{C},(c) 1325^{\circ} \mathrm{C}$ и $(d) 1350^{\circ} \mathrm{C}$.

температурах близких к комнатным. Зависимость изменения температуры $\Delta T$ при ЭКЭ приведена на рис. 6. Различия в зависимости $\Delta T$ для процессов поляризации и деполяризации объясняется наличием пиротоков [30].

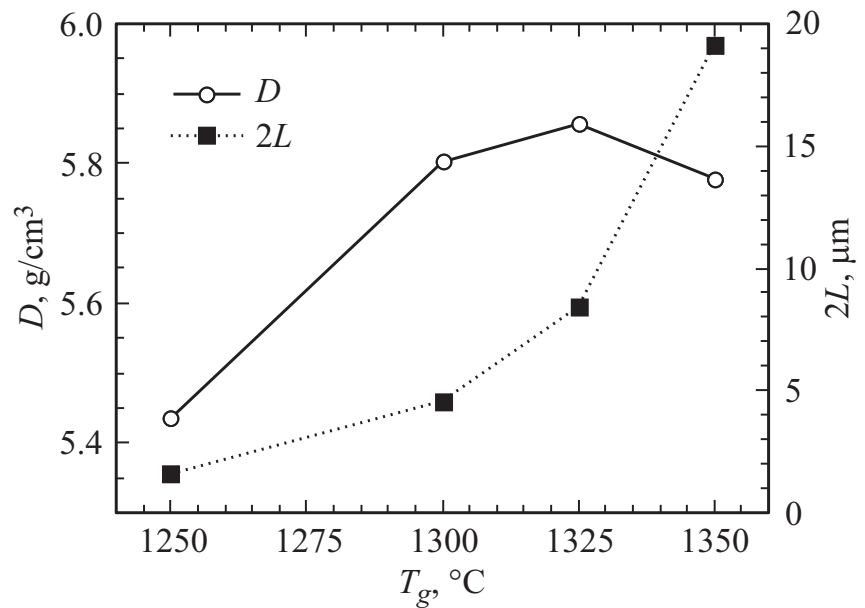

Рис. 4. Зависимость плотности $D$ и среднего размера зерен $L$ от температуры обжига синтеза $T_{\mathrm{g}}$.

\section{4. Обсуждение результатов}

Физические процессы во время обжига керамики описываются следующим образом. Изначальный порошок состоит из гранул, имеющих форму шара, между которыми имеются поры. При росте температуры синтеза увеличивается как средний размер гранул, так и наибольший. Как следствие этого, уменьшается площадь поверхности зерен. При увеличении температуры обжига выше $1300^{\circ} \mathrm{C}$ свойства керамики начинают резко меняться. При $1325^{\circ} \mathrm{C}$ плотность керамики принимает наибольшее значение (рис. 2). Наиболее сильное изменение в размере гранул происходит в промежутке от $1300^{\circ} \mathrm{C}$ до $1350^{\circ} \mathrm{C}$. Аналогичный эффект изменения скорости роста гранул в указанном температурном диапазоне наблюдался в $[6,16]$. При этом заметно увеличивается ЭКЭ. Так, для керамики, отожженной при $1350^{\circ} \mathrm{C}$, ЭКЭ в 4 раза больше, чем у керамики с $T_{\mathrm{g}}=1300^{\circ} \mathrm{C}$ (рис. 6). Увеличивается также диэлектрическая проницаемость $\varepsilon$ и потери $(\tan \delta)$ (рис. 5). Подобное явление отмечалось в работе [6] и связывалось с изменением при $1320^{\circ} \mathrm{C}$ знака асимметрии у распределения гранул по размерам. Это 

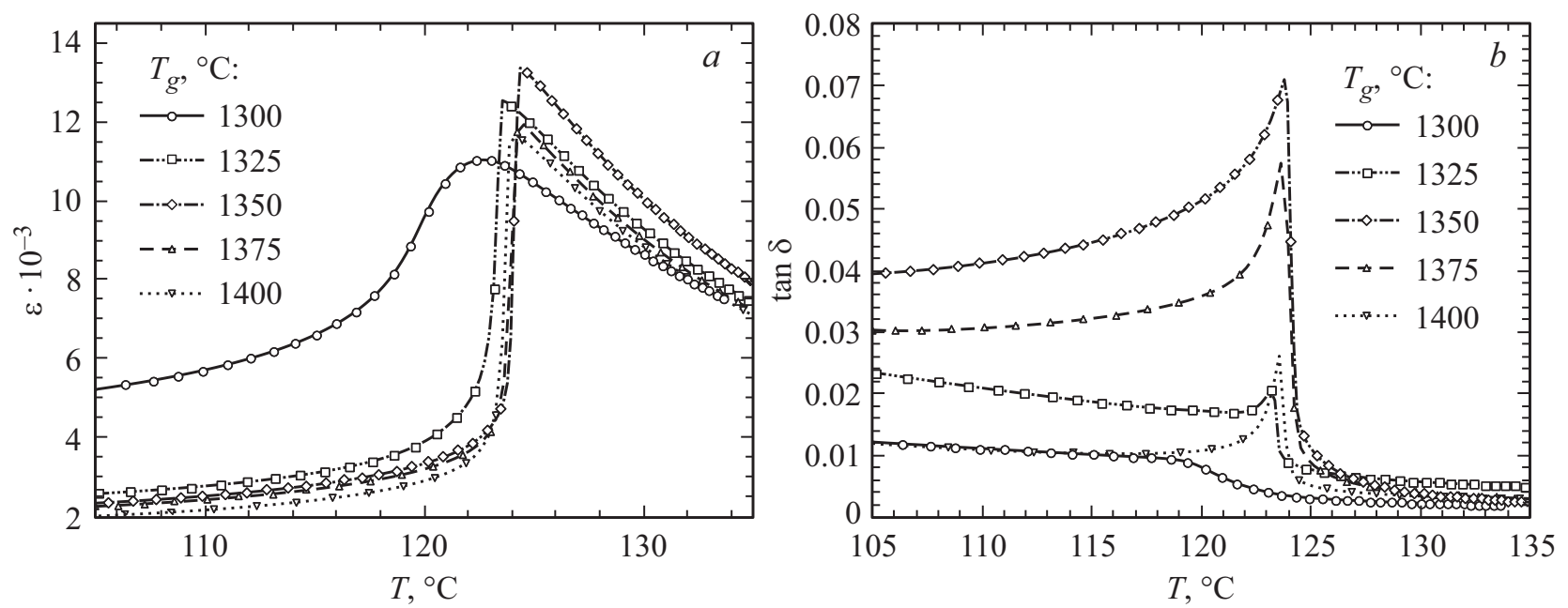

Рис. 5. Температурная зависимость $(a)$ диэлектрической проницаемости $\varepsilon$ и $(b)$ диэлектрических потерь $\tan \delta$ для различных температур $T_{\mathrm{g}}$.
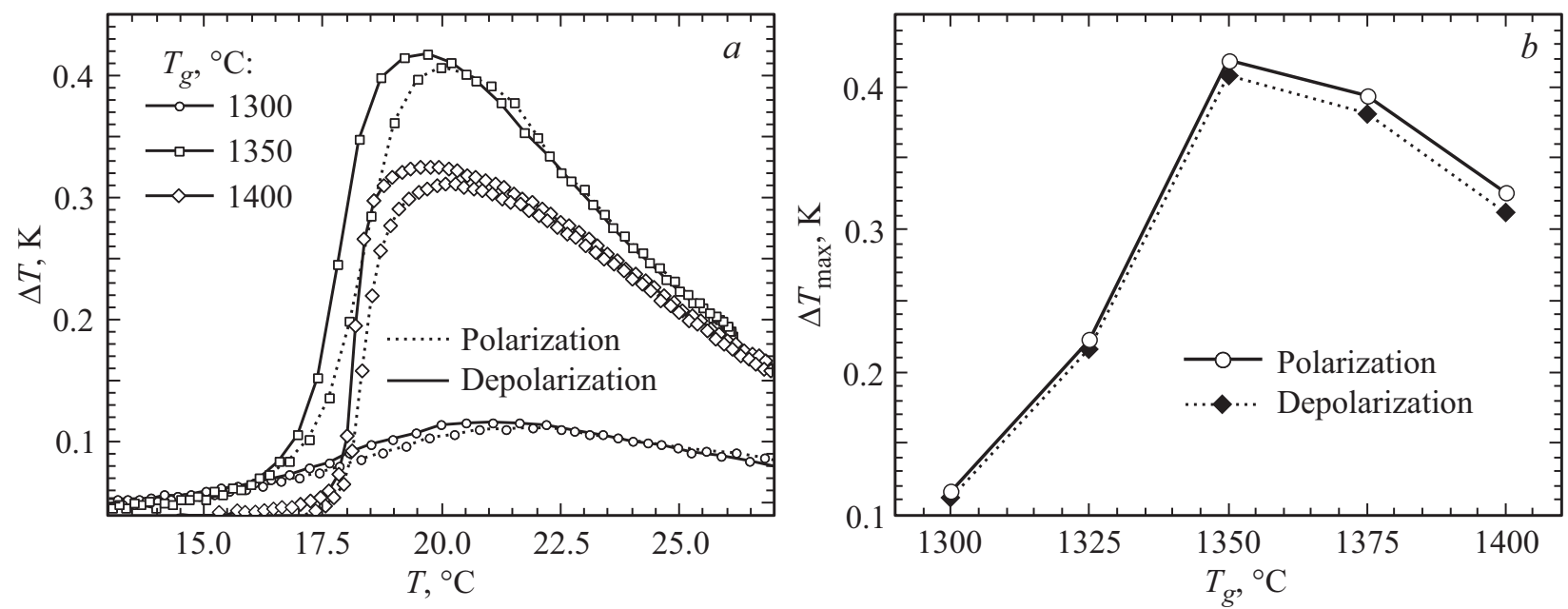

Рис. 6. (a) Зависимость изменения температуры $\Delta T$ при электрокалорическом эффекте от температуры $T$ для образцов, отожженных при различных режимах $T_{\mathrm{g}}$. $(b)$ Зависимость наибольшего изменения температуры $\Delta T_{\max }$ при ЭКЭ от температуры обжига синтеза.

означает, что при $T>1320^{\circ} \mathrm{C}$ число гранул с размерами, превышающими математическое ожидание, превосходит число гранул с меньшими размерами. В соответствии с вышеизложенной теорией диэлектрическая проницаемость и ЭКЭ растут с увеличением размера гранул. Температура фазового перехода $T_{\mathrm{PT}}$ в соответствии с формулой (18) также является монотонно возрастающей функцией как размера гранулы, так и плотности керамики (рис. 7,a). Отметим, что при сравнении с экспериментальными данными нужно учитывать не только изменение размера гранулы, но и плотность керамики. Для оценки влияния свойств диэлектрического слоя были построены зависимости температуры $T_{\mathrm{PT}}$ от значений коэффициентов упругости (рис. 7, $b$ ) и толщины (рис. 7,c) диэлектрического слоя. При проведении численных расчетов упругие постоянные диэлектрического слоя $c_{j k}^{1}$ полагались пропорциональ- ными упругим коэффициентам шара $c_{j k}^{i}$. Полученные зависимости однозначно указывают, что для керамики с размером гранул, превышающим $1 \mu \mathrm{m}$, температура фазового перехода практически не зависит ни от упругих свойств диэлектрического слоя, ни от его толщины. Влияние диэлектрического слоя на $T_{\mathrm{PT}}$ становится заметной только для гранул с $R<0.5 \mu \mathrm{m}$.

Уменьшение плотности керамики, согласно уравнению (19), может приводить к наличию максимума зависимости температуры фазового перехода в керамике $T_{\mathrm{PT}}$ от температуры обжига синтеза. Для проверки данного утверждения был построен график зависимости $T_{\mathrm{PT}}$ и температуры максимума диэлектрической проницаемости $T_{\mathrm{m}}$ от $T_{\mathrm{g}}$ (рис. 8). Зависимость $T_{\mathrm{PT}}\left(T_{\mathrm{g}}\right)$ находилась по температурной зависимости $\varepsilon$ на основании закона Кюри. Для сравнения также приведены результаты расчета $T_{\mathrm{PT}}$ на основе (18) в рамках четырехфазной 

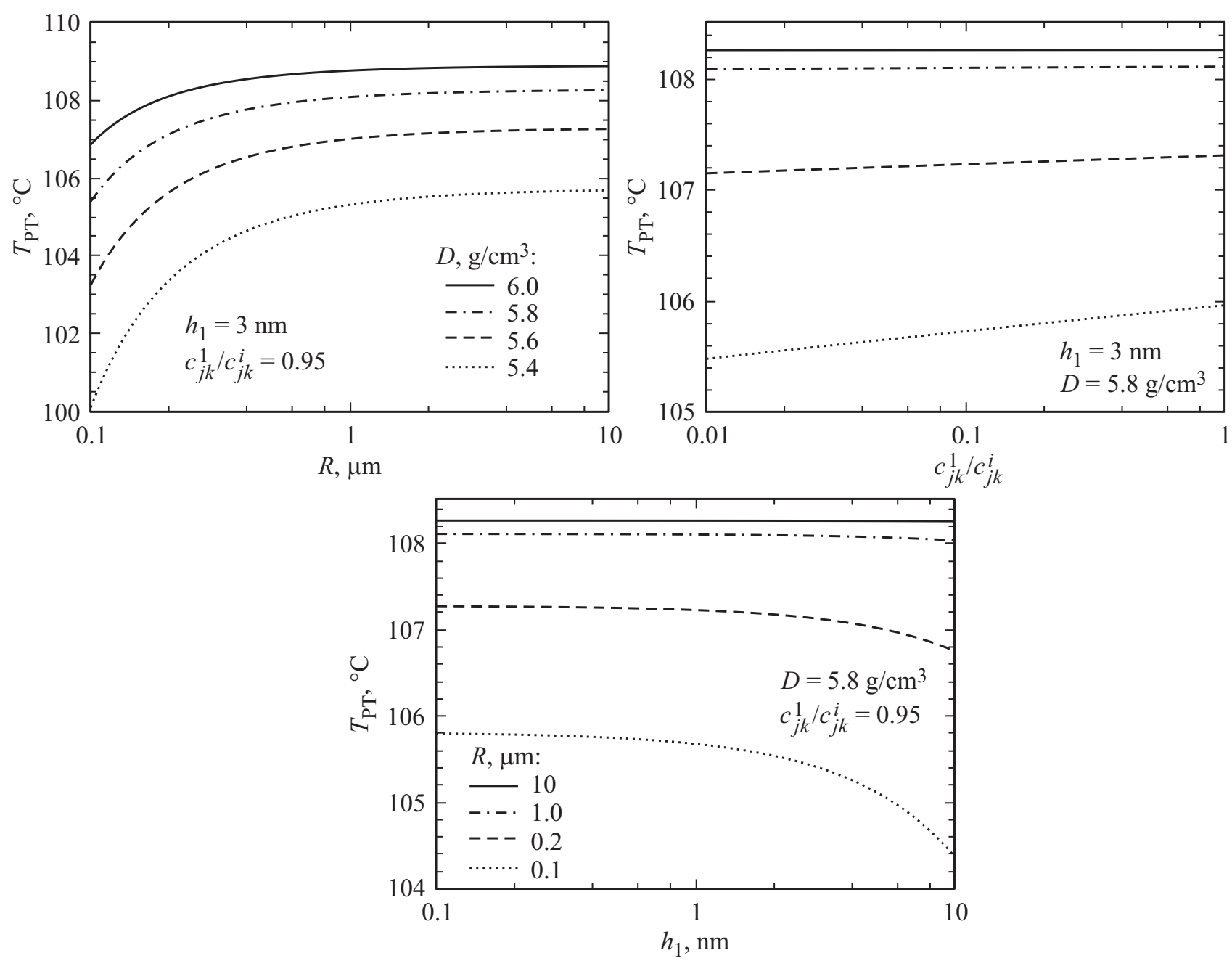

Рис. 7. Зависимость температуры фазового перехода от размера гранулы при различных значениях плотности (a), упругих свойств $(b)$ и толщины диэлектрического слоя при различных размерах гранулы $(c)$.

модели с использованием данных из [22]. Максимальное значение $T_{\mathrm{PT}}$ достигается при $1350^{\circ} \mathrm{C}$ в полном соответствии с теорией. Существование максимума $T_{\mathrm{PT}}$ вызвано свойствами воздушного слоя и, вследствие этого, неустойчиво по отношению к изменению условий синтеза керамики.

Наивысшими значениями диэлектрической проницаемости и температуры фазового перехода, а также наибольшим ЭКЭ при комнатных температурах, обладает керамика с $T_{\mathrm{g}}=1350^{\circ} \mathrm{C}$. Для керамик с другими температурами обжига вышеперечисленные величины ведут себя независимо друг от друга (см. рис. 5,6,8). Отсутствие сильной корреляции между диэлектрической проницаемостью и ЭКЭ вызвано тем обстоятельством, что ЭКЭ определяется производной от поляризации по температуре (пироэлектрическим коэффициентом П). Тем не менее, связь между этими величинами имеется, так как увеличение диэлектрической проницаемости ведет к увеличению поляризации, и, следовательно, к увеличению П. При росте температуры обжига син-

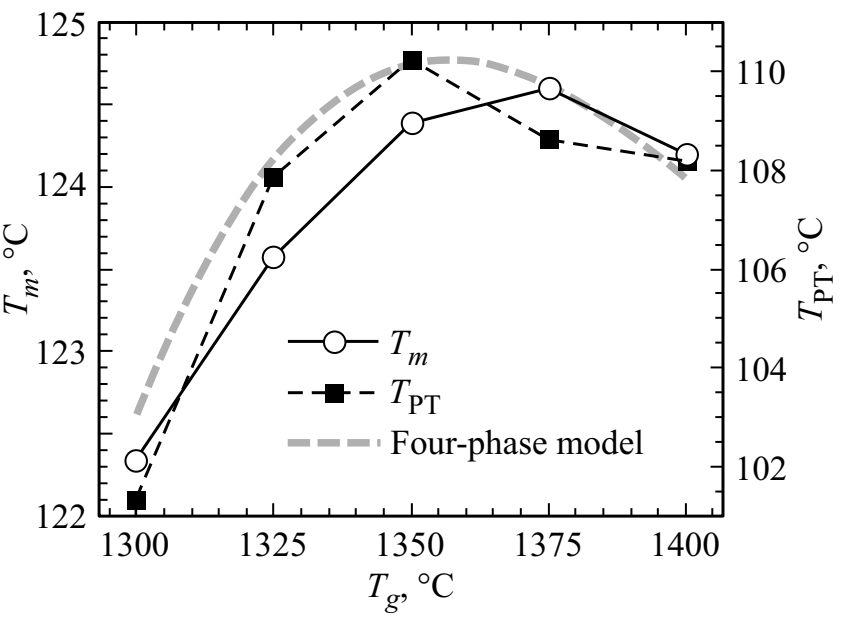

Рис. 8. Зависимость температуры максимума диэлектрической проницаемости $T_{\mathrm{m}}$ и температуры фазового перехода $T_{\mathrm{PT}}$ от температуры обжига синтеза. Пунктирной линией представлены результаты четырехфазной модели для $T_{\mathrm{PT}}$. 
теза выше $1350^{\circ} \mathrm{C}\left(1365^{\circ} \mathrm{C}\right.$ согласно [6]) происходит уменьшение ЭКЭ и диэлектрической проницаемости. На наш взгляд это связано с двумя обстоятельствами. При $1350-1365^{\circ} \mathrm{C}$ распределение гранул имеет небольшую дисперсию. При увеличении температуры или времени обжига синтеза дисперсия увеличивается вследствие диффузионного характера процесса обжига [31]. Распределение зерен по размеру все дальше уходит от $\delta$-образного и становится все ближе к равномерному. Для среднего размера зерен в $25 \mu \mathrm{m}$ имеется некоторое количество зерен с размером $50 \mu \mathrm{m}$ и некоторое количество зерен размера меньшего $200 \mathrm{~nm}$. Температура фазового перехода для зерен крайних размеров отличается на $20^{\circ} \mathrm{C}$ и более. В результате происходит размытие острого пика зависимости ЭКЭ от температуры. Аналогичным образом объясняется „сплющивание“ температурной зависимости диэлектрической проницаемости и уменьшение ее максимального значения. Кроме того, уменьшается площадь зерен, но не уменьшается объем пор. Это означает, что свойства переходного слоя становятся ближе к свойствам пор. Его диэлектрическая проницаемость и ЭКЭ уменьшаются. Два указанных обстоятельства и приводят к существованию максимумов диэлектрической проницаемости и ЭКЭ при определенном размере гранул.

\section{5. Заключение}

В работе предложено рассматривать керамику как композитную среду, в которой включения имеют форму шара, покрытого диэлектрической и воздушной оболочкой (четырехфазная среда). Свойства воздушного слоя, моделирующего поры в керамике, сильно зависят от условий синтеза. Наоборот, свойства диэлектрического слоя практически неизменны. В предположении об однородности поляризации удалось решить упругую задачу для одиночной гранулы и найти коэффициенты в уравнении Ландау-Гинзбурга. Учет упругости приводит к появлению зависящих от радиуса слагаемых в коэффициентах ЛГ. Изменение коэффициента $a$ пропорционально давлению $p$, с которым на данную гранулу воздействуют соседние. Из-за отличия коэффициентов $a$ и $\hat{a}$ следует выведенный ранее из экспериментов закон убывания температуры Кюри-Вейсса с уменьшением размера гранулы, а также существование максимума в зависимости температуры фазового перехода от размера гранул. Постоянная, определяющая закон убывания температуры Кюри-Вейсса, определяется, в основном, свойствами диэлектрического слоя и поэтому слабо зависит от условий эксперимента. Наличие же температуры обжига $T_{\mathrm{g}}$, при которой температура Кюри-Вейсса имеет максимум, определяется свойствами воздушного слоя, который весьма чувствителен к качеству исходного порошка и условиям обжига. Вследствие этого данный эффект нестабилен и не всегда может проявляться. Наличие зависящих от радиуса слагаемых в коэффици- енте $\hat{b}$, которые возникают благодаря электрострикции, позволяет объяснить изменение температур фазовых переходов между тетрагональной, орторомбической и ромбоэдрической фазами. В отличие от температуры фазового перехода между кубической и тетрагональной фазами (Кюри-Вейсса) эти температуры с ростом размера гранул могут уменьшаться [32]. Данное явление не может быть объяснено в рамках стандартной модели [19], где от размера гранул зависит только коэффициент $\hat{a}$.

Важную роль в электрических и термодинамических свойствах сегнетоэлектрической керамики играет функция распределения зерен по размеру и свойства переходного слоя, в которых учитывается наличие пор. Для получения наибольшего значения величины ЭКЭ следует получать зерна примерно одинаковых размеров. Полученное в эксперименте максимальное значение ЭКЭ для керамики титаната бария при комнатной температуре, равное $0.42 \mathrm{~K}$, превосходит ранее известные [6-9]. Этот результат показывает превосходство метода двуступенчатого синтеза. Увеличение температуры синтеза $T_{\mathrm{g}} \mathrm{c}$ 1300 до $1350^{\circ} \mathrm{C}$ ведет к увеличению ЭКЭ $\Delta T$ в 4 раза, и примерно в такой же пропорции увеличивается средний размер гранул. Дальнейшее повышение $T_{\mathrm{g}}$ приводит к уменьшению ЭКЭ, но средний размер гранул при этом увеличивается. Значит, существует оптимальный размер гранул, при котором ЭКЭ достигает наибольшего значения. Разработанная в работе теория также указывает на существование такого размера. К сожалению, оптимальный размер гранул сильно зависит от начального размера гранул порошка и от условий синтеза. С определенностью можно сказать, что наногранулированные пленки $(40 \mathrm{~nm}<L<1 \mu \mathrm{m})$ обладают меньшим ЭКЭ, чем микрогранулированные $(L>1 \mu \mathrm{m})$, и оптимальное значение размера гранул может варьироваться от нескольких микрон до десятков микрон. В данной работе наибольшим ЭКЭ обладает керамика с размером гранул $L \approx 10 \mu \mathrm{m}$. Отсюда следует вывод, что коммерческие многослойные керамические конденсаторы не имеет смысла использовать в охлаждающих устройствах, так как размер гранул в них выбран для получения наилучшей управляемости или наибольшей диэлектрической проницаемости $(L \sim 1 \mu \mathrm{m})$. Экспериментальные данные настоящей работы ясно показывают наличие сильной зависимости тепловых и электрических свойств сегнетоэлектрической керамики от температуры и времени синтеза. Разработанная теоретическая модель позволяет описывать эти свойства количественно и может быть использована для оптимизации ЭКЭ и создания высокоэффективного твердотельного охладителя.

\section{Финансирование работы}

Исследование выполнено за счет гранта Российского научного фонда (проект № 19-79-10074). 
Электронно-микроскопические исследования выполнены с использованием оборудования федерального ЦКП „Материаловедение и диагностика в передовых технологиях“, поддержанного Минобрнауки России.

\section{Конфликт интересов}

Авторы заявляют об отсутствии конфликта интересов.

\section{Список литературы}

[1] А.С. Сигов, Е.Д. Мишина, В.М. Мухортов. ФТТ 52, 4, 709 (2010).

[2] A. Starkov, O. Pakhomov, I. Starkov. Ferroelectrics 430, 1, 108 (2012).

[3] S. Karmanenko, A. Semenov, A. Dedyk, A. Es'kov, A. Ivanov, P. Beliavskiy, Yu. Pavlova, A. Nikitin, I. Starkov, A. Starkov, O. Pakhomov. New Approaches to Electrocaloric-Based Multilayer Cooling. In the book Electrocaloric Materials. Springer, Berlin Heidelberg (2014) Ch. VIII, P. 183-223.

[4] B.M. Vul, I.M. Goldman. Compt. Rend. Acad. Sci. URSS 49, 177 (1945).

[5] Y. Bai, K. Ding, G.P. Zheng, S.Q. Shi, L. Qiao. Phys. Status Solidi A 209, 5, 941 (2012).

[6] B.C. Kim, K.W. Chae, C.I Cheon. J. Korean Phys. Soc. 76, 3, $226(2020)$

[7] X.C. Ren, W.L. Nie, Y. Bai, L.J. Qiao. Eur. Phy. J. B 88, 9, 1 (2015).

[8] А.В. Карташев, В.С. Бондарев, И.Н. Флёров, М.В. Горев, Е.И. Погорельцев, А.В. Шабанов, М.С. Молокеев, S. Guillemet-Fritsch, И.П. Раевский. ФТТ 61, 6, 1128 (2019).

[9] S. Patel, M. Kumar. AIP Advances 10, 8, 085302 (2020).

[10] M.T. Buscaglia, M. Viviani, V. Buscaglia, L. Mitoseriu, A. Testino, P. Nanni, Z. Zhao, M. Nygren, C. Harnagea, D. Piazza, C. Galassi. Phys. Rev. B 73, 064114 (2006).

[11] T. Hoshina, S. Wada, Y. Kuroiwa, T. Tsurumi. Appl. Phys. Lett. 93, 192914 (2008).

[12] B.A. Strukov, S.T. Davitadze, S.G. Shulman, B.V Goltzman, V.V. Lemanov. Ferroelectrics 301, 1, 157 (2004).

[13] N.A. Pertsev, A.G. Zembilgotov, A.K. Tagantsev. Phys. Rev. Lett. 80, 9, 1988 (1998).

[14] P. Zheng, J.L. Zhang, Y.Q. Tan, C.L. Wang. Acta Mater. 60, 13-14, 5022 (2012).

[15] M.V. Zdorovets, A.L. Kozlovskiy. Vacuum 168, 108838 (2019).

[16] O.V. Malyshkina, G.S. Shishkov, A.A. Martyanov, A.I. Ivanova. Mod. Electron. Mater. 6, 141 (2020).

[17] О.Г. Вендик, Н.Ю. Медведева, С.П. Зубко. ПжТФ 34, 8, 13 (2008).

[18] A.S. Starkov, I.A. Starkov, A.I. Dedyk, G. Suchaneck, G. Gerlach. Phys. Status Solidi B 255, 2, 1700245 (2018).

[19] J.H. Qiu, Q. Jiang. J. Appl. Phys. 105, 3, 034110 (2009).

[20] L. Wu, M.C. Chure, K.K. Wu, W.C. Chang, M.J. Yang, W.K. Liu, M.J. Wu. Ceram. Int. 35, 3, 957 (2009).

[21] Q. Jin, B. Cui, X. Zhang, J. Wang. J. Electron. Mater. 50, 1, 325 (2021).

[22] P. Marton, I. Rychetsky, J. Hlinka. Phys. Rev. B 81, 14, 144125 (2010).
[23] Дж. Най. Физические свойства кристаллов и их описание при помощи тензоров и матриц. Изд-во ИЛ, М. (1960). $377 \mathrm{c}$.

[24] Л.Д. Ландау, Е.М. Лифшиц. Теория упругости. Наука, М. (1987).

[25] А.С. Старков, И.А. Старков. ЖЭТФ 146, 5, 980 (2014).

[26] M. Vrabelj, H. Uršič, Z. Kutnjak, B. Rožič, S. Drnovšek, A. Benčan, V. Bobnar, L. Fulanovič, B. Malič. J. Eur. Ceram. Soc. 36, 75 (2016).

[27] S. Hu, C. Luo, P. Li, J. Hu, G. Li, H. Jiang, W. Zhang. J. Mater. Sci. Mater. El. 28, 13, 9322 (2017).

[28] W. Heywang. J. Mater. Sci. 6, 1214 (1971).

[29] N. Funsueb, A. Limpichaipanit, A. Ngamjarurojana. J. Phys.: Conf. Ser. 1144, 1, 012133 (2018).

[30] A. Starkov, I. Starkov, Ferroelectrics 461, 1, 50 (2014).

[31] Л.Д. Ландау, Е.М. Лифшиц. Гидродинамика. Наука, М. (1986).

[32] X.H. Wang, I.W. Chen, X.Y. Deng, Y.D. Wang, L.T. Li. J. Adv. Ceram. 4, 1, 1 (2015).

Редактор Т.Н. Василевская 